\title{
NAT-rock formation by mother clouds: a microphysical model study
}

\author{
S. Fueglistaler ${ }^{1}$, B. P. Luo ${ }^{1}$, C. Voigt ${ }^{1}$, K. S. Carslaw ${ }^{2}$, and Th. Peter ${ }^{1}$ \\ ${ }^{1}$ Atmospheric and Climate Science, ETH Zürich, Switzerland \\ ${ }^{2}$ School of the Environment, University of Leeds, Leeds, UK
}

Received: 11 December 2001 - Published in Atmos. Chem. Phys. Discuss.: 14 January 2002

Revised: 19 April 2002 - Accepted: 29 April 2002 - Published: 15 May 2002

\begin{abstract}
Polar stratospheric clouds (PSCs) of type 1a or 1aenh containing high number densities of nitric acid trihydrate (NAT) particles, can act as mother clouds for extremely large NAT particles, termed NAT-rocks, provided the air below the clouds is supersaturated with respect to NAT. Individual NAT particles at the cloud base fall into undepleted gas phase and rapidly accelerate due to a positive feedback between their growth and sedimentation. The resulting reduction in number density is further enhanced by the strong $\mathrm{HNO}_{3}$ depletion within a thin layer below the mother cloud, which delays subsequent particles. This paper introduces the basic microphysical principles behind this mother cloud/NAT-rock mechanism, which produces $10^{-4} \mathrm{~cm}^{-3}$ NAT-rocks with radii around $10 \mu \mathrm{m}$ some kilometers below the mother cloud. The mechanism does not require selective nucleation and works even for a monodisperse particle size distribution in the mother cloud.
\end{abstract}

\section{Introduction}

Denitrification of the Arctic stratospheric vortex is believed to enhance halogen-catalyzed chemical reactions leading to ozone depletion (WMO, 1999; Waibel et al., 1999; Tabazadeh et al., 2001; Gao et al., 2001). A major finding of the SOLVE/THESEO-2000 campaign is the widespread occurrence of large nitric acid hydrate particles of up to $10 \mu \mathrm{m}$ in radius in extremly low number densities $\left(n \approx 10^{-4} \mathrm{~cm}^{-3}\right)$ (Fahey et al., 2001). Most likely these large particles were composed of NAT $\equiv \mathrm{HNO}_{3} \cdot 3 \mathrm{H}_{2} \mathrm{O}$ (commonly called "NAT-rocks" [Fahey, pers. comm.]). They are mostly out of thermodynamic equilibrium with the ambient gas phase due to their low number densities, which leads to long particle/gas phase equilibration times. In turn, this allows the

Correspondence to: S. Fueglistaler

(sf@atmos.umnw.ethz.ch) particles to grow to the observed sizes which enable them to sediment rapidly and thus to efficiently denitrify the corresponding layers of the polar stratosphere.

PSCs of type 1a and 1a-enh, presumably consisting of NAT, have been extensively studied for several years (Browell et al., 1990; Toon et al., 1990; Tsias et al., 1999; Toon et al., 2000; Voigt et al., 2000). While 1a-enh PSCs have typical number densities $n \approx 0.1 \mathrm{~cm}^{-3}$ and are therefore close to thermodynamic equilibrium with the ambient gas phase, type 1a PSCs have lower particle number densities and longer equilibration times ( $\gtrsim 1$ day) (Biele et al., 2001). NAT clouds with $n \gtrsim 10^{-2} \mathrm{~cm}^{-3}$ have limited particle radii $r \lesssim 2.5 \mu \mathrm{m}$. Such particles have only small sedimentation velocities and thus cannot lead to efficient denitrification. Here we show that dense NAT clouds can be efficient sources for NAT-rocks (a similar mechanism is suggested by, Dhaniyala et al., 2002) explaining their observed characteristics with respect to particle number density and size without highly selective nucleation, e.g. homogeneous freezing of NAT (Tabazadeh et al., 2001).

\section{The basic mechanism}

In this study a mechanism is proposed where NAT clouds with high particle number densities $\left(n \gtrsim 0.01 \mathrm{~cm}^{-3}\right)$ act as "mother clouds", that is, as efficient sources for NAT-rocks via sedimentation. Mother clouds are close to thermodynamic equilibrium, i.e. the saturation ratio $S_{\mathrm{NAT}}$ of $\mathrm{HNO}_{3}$ with respect to NAT within the cloud is close to unity. However, below the cloud where NAT particles are absent or extremly low in number density, $S_{\mathrm{NAT}}$ may be larger than 1 (see e.g. Fueglistaler et al., 2002, for observations). NAT particles that sediment out of the mother cloud fall into this supersaturated air, begin to grow and hence accelerate. In this paper we show results of a model that solves the coupled equations for particle growth, sedimentation and gas phase depletion. 

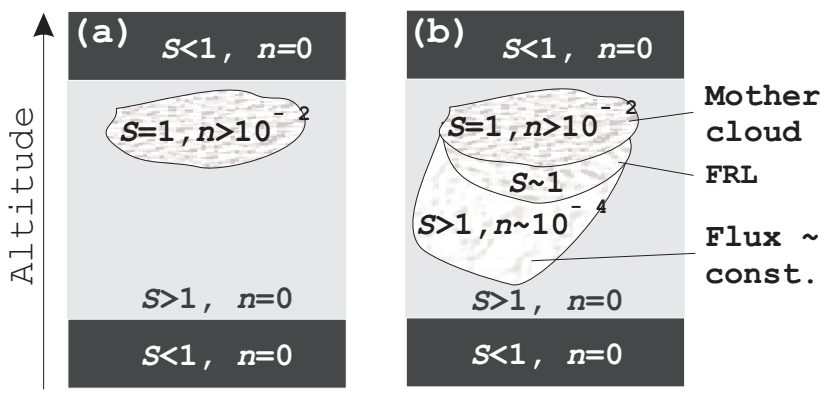

Fig. 1. Schematic vertical cross section of NAT particle sedimentation out of a mother cloud floating in supersaturated air. $S$ is the saturation ratio with respect to NAT, $n$ the NAT particle number density. (a) A few hours after mother cloud formed. (b) A few days later after NAT particles have sedimented out. The flux reduction layer (FRL) is shown schematically. Sizes in drawing not in proportion, deformation in (b) sketches deformation caused by wind shear.

However, we will first present a theoretical discussion to gain insight into the suggested mechanism.

To break down the complex interplay of particle growth, acceleration and gas phase depletion, we first consider the hypothetical case where the growing particles do not deplete the gas phase. All particles then experience the same growth and sedimentation once they leave the mother cloud. The sedimentation velocity $v_{\text {sed }}$ of a particle can then be expressed as a function of altitude $z$ alone. This stationary solution conserves the particle number flux $j_{\mathrm{p}}$ (i.e. the sedimentation velocity, particle number density and particle number flux are functions of the altitude but do not explicitly depend on time):

$j_{\mathrm{p}}(z)=n(z) \cdot v_{\mathrm{sed}}(z)=$ const.

Since $v_{\text {sed }}$ is roughly proportional to the square of the particle radius $r$ (Müller and Peter, 1992), the particle acceleration due to growth alone, i.e. ignoring gas phase depletion, is able to significantly reduce the particle number density. For a dense mother cloud with $n=1 \mathrm{~cm}^{-3}$ an increase in radius from $1 \mu \mathrm{m}$ (the approximate NAT particle radius in the mother cloud in thermodynamic equilibrium) to $10 \mu \mathrm{m}$ leads to a reduction of the particle number density by two orders of magnitude.

The particle number flux will not remain constant when $\mathrm{HNO}_{3}$ gas phase depletion is taken into account. The falling NAT particles remove $\mathrm{HNO}_{3}$ from the gas phase, and subsequent particles (falling through depleted air) will grow more slowly and hence accelerate less readily, leading to a decrease in the particle flux. We show below that this effect controls the particle flux in the vicinity of the cloud base, but becomes less important with increasing distance from the mother cloud, where particles fall rapidly and therefore denitrify less.
Thus, there are two particle flux regimes: in the vicinity of the cloud base, particle number flux is reduced by the $\mathrm{HNO}_{3}$ depletion by the sedimenting particles, here termed flux reduction layer (FRL). In contrast, sufficiently far below the mother cloud the flux is approximately conserved (Fig. 1).

\section{Description of the microphysical model}

We use a 1-D microphysical model to study the physical processes taking place at the base of a mother cloud. The gas phase is modeled on an irregularly spaced Eulerian grid with 8800 points. Centered at the cloud base, 8000 points are used to model this important region with a vertical resolution of $\Delta z=1 \mathrm{~cm}$. Elsewhere the vertical resolution is $10 \mathrm{~m}$, covering a column from $15 \mathrm{~km}$ to $23 \mathrm{~km}$ altitude.

The NAT particles are modelled as individual particles, storing particle positions and radii. About 50000 particles were required to obtain a statistically sufficient number of particles at all altitude levels. The NAT particle size distribution in the mother cloud may be freely prescribed and is chosen to be monodisperse for the base line calculation, with the radius determined from the particle number density in the mother cloud and the available $\mathrm{HNO}_{3}$ in thermodynamic equilibrium. For each timestep $(\Delta t=30 \mathrm{~s}) \mathrm{HNO}_{3}$ partitioning between the gaseous and condensed phases is calculated from the kinetic growth/evaporation equations for the NAT particles and by assuming equilibrium between the supercooled ternary solution (STS) droplets and the gas phase. The $\mathrm{HNO}_{3}$ vapour pressure of NAT is calculated according to Hanson and Mauersberger (1988), the partitioning into STS according to Carslaw et al. (1995). Particle sedimentation velocities are calculated including the Stokes and slip-flow regimes (Müller and Peter, 1992). The shape of the particles undergoing growth/evaporation and sedimentation may be prescribed and is chosen to be spherical in the base line case.

The model was initialized with a constant $\mathrm{H}_{2} \mathrm{O}$ volume mixing ratio (VMR) of 5 ppmv and a constant total $\mathrm{HNO}_{3}$ VMR of 8 ppbv (typical for SOLVE/THESEO-2000 midwinter conditions). The time-independent temperature profile is chosen such that initially $S_{\mathrm{NAT}}=10$ in the entire column. This corresponds to a temperature of $\approx 3 \mathrm{~K}$ below $\mathrm{T}_{\mathrm{NAT}}$ and a negligible $\mathrm{HNO}_{3}$ partitioning into STS (less than $1 \%$ for $0.1 \mathrm{ppbv}_{2} \mathrm{SO}_{4}$ ). In the mother cloud, NAT particles are then allowed to reduce the saturation ratio to $S_{\mathrm{NAT}}=1$. These idealized conditions have been chosen to study the mechanism without effects caused by complex gas phase and temperature profiles.

\section{Results}

Model calculations have been performed for a wide range of cloud and gas phase parameters. Figure 2 shows the detailed results for a monodisperse mother cloud with $n=0.1 \mathrm{~cm}^{-3}$ 

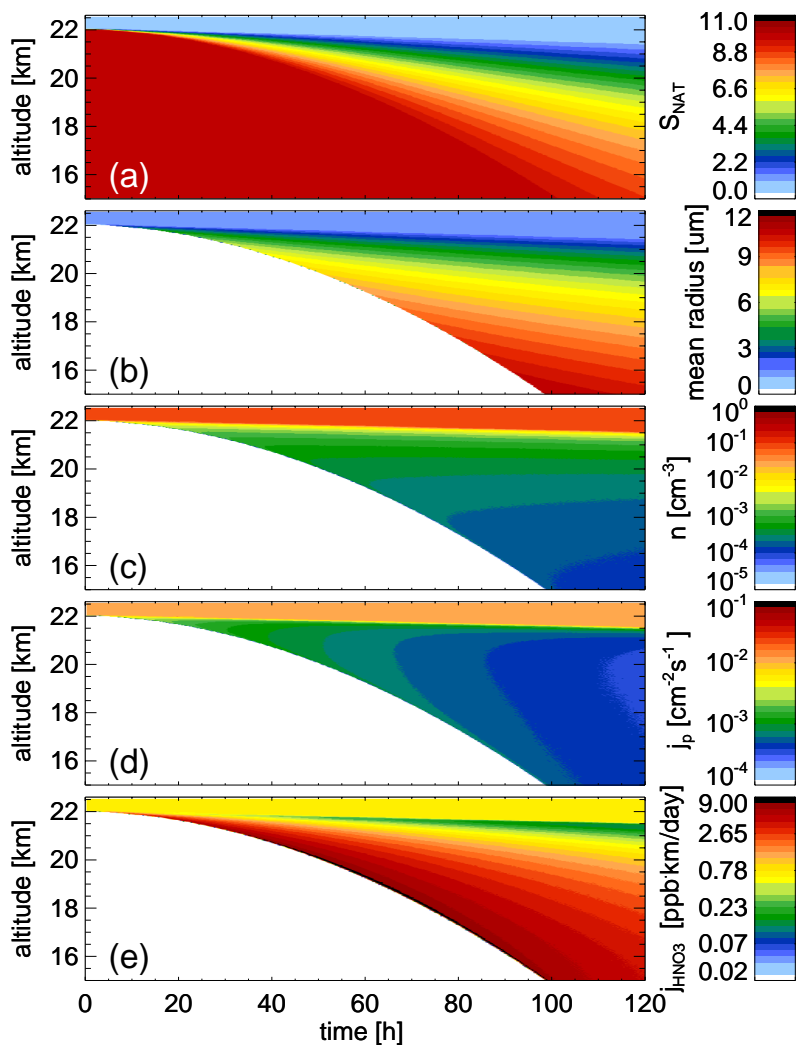

Fig. 2. 1-D model results for base line case as described in text. (a) Saturation ratio $S_{\mathrm{NAT}}(z, t)$ with $S_{\mathrm{NAT}}=1$ in the mother cloud and $>1$ below. (b) - (e) Resulting radius $r(z, t)$, number density $n(z, t)$ particle flux $j_{p}(z, t)$ and $\mathrm{HNO}_{3}$ mass flux $j_{\mathrm{HNO} 3}(z, t)$. The minimum in the $\mathrm{HNO}_{3}$ flux (panel e) just below the mother cloud base is a direct consequence of the strong particle number flux reduction in this region (the Flux Reduction Layer). The total $\mathrm{HNO}_{3}$ is shown in Fig. 4a.

(typical for type 1a-enh) and standard parameters mentioned above.

The flux reduction layer (FRL), due to strong $\mathrm{HNO}_{3}$ depletion below the cloud base, causes an impressive reduction in both particle number density (c) and flux (d) by 2 orders of magnitude. Panel (e) shows the $\mathrm{HNO}_{3}$ mass flux. A flux of $1 \mathrm{ppb} \times \mathrm{km} /$ day corresponds to $1 \mathrm{ppb} \mathrm{HNO}_{3}$ being transported downward by $1 \mathrm{~km}$ within one day. The $\mathrm{HNO}_{3}$ mass flux due to NAT-rocks reaches up to $9 \mathrm{ppb} \times \mathrm{km} /$ day, while it is $0.8 \mathrm{ppb} \times \mathrm{km} /$ day within the mother cloud and remains limited to the corresponding small altitude range.

A quantification of the effect of gas phase depletion, caused by the sedimenting particles, on particle number density is given in Fig. 3. Particle number density profiles for the base line case, a more dense $\left(n=1 \mathrm{~cm}^{-3}\right.$, all other parameters as in base line case) and a less dense $\left(n=0.01 \mathrm{~cm}^{-3}\right.$, all other parameters as in base line case) mother cloud are compared to their corresponding stationary solution. Three conclusions follow from this comparison: (1) The decrease of

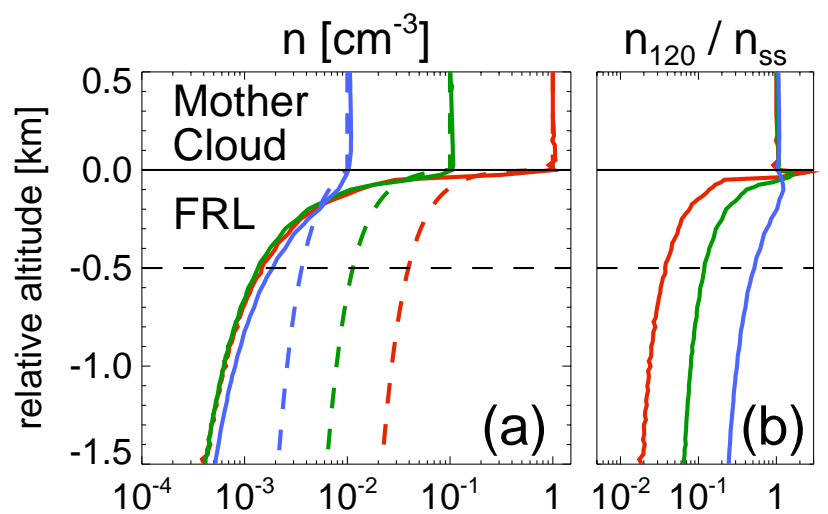

Fig. 3. (a) Vertical profiles of particle number density without gas phase depletion (stationary solution, dashed lines) and with gas phase depletion (full model, solid lines). All calculations use the same parameters as the baseline case shown in Fig. 2, except for the particle number density in the mother cloud (red: $n=1 \mathrm{~cm}^{-3}$, $r_{\mathrm{N} A T}^{\text {equil }} \approx 0.6 \mu \mathrm{m}$; green: $n=10^{-1} \mathrm{~cm}^{-3}, r_{\mathrm{N} A T}^{\text {equil }} \approx 1.25 \mu \mathrm{m}$; blue: $\left.n=10^{-2} \mathrm{~cm}^{-3}, r_{\mathrm{N} A T}^{\text {equil }} \approx 2.7 \mu \mathrm{m}\right)$. The model results show the number density profile after 5 days $(120 \mathrm{~h})$. The stationary calculations use the same initial conditions as the corresponding model calculations but neglect gas phase depletion. (b) The number density of the model calculations after 5 days $\left(n_{120}\right)$ divided by the number density of the corresponding stationary solution $\left(n_{\mathrm{s} s}\right)$. This ratio allows a quantification of the effect of the gas phase depletion on particle number density reduction. The effect is most prominent within a thin layer below the mother cloud, the flux reduction layer (FRL).

particle number density due to gas phase depletion and due to particle acceleration are of the same order of magnitude; (2) The effect of gas phase depletion on particle number density reduction is most prominent in a relatively thin layer just below the mother cloud, the flux reduction layer (FRL); (3) The combination of particle acceleration and gas phase depletion leads to particle number densities around $10^{-4} \mathrm{~cm}^{-3}$ a few kilometers below the mother cloud, independently of the particle number density in the mother cloud. Hence, the suggested mother cloud/NAT-rock mechanism produces the observed NAT-rock particle number densities, $n \approx 10^{-4} \mathrm{~cm}^{-3}$, from the whole range of mother cloud particle number densities.

The resulting total $\mathrm{HNO}_{3}$ VMR (gas plus condensed phase) of the base line case is shown in panel (a) of Fig. 4, allowing a quantification of denitrification. The most severe denitrification is found in the FRL, where it reaches up to $75 \%$. After 5 days, a vertical layer of about $2 \mathrm{~km}$ is denitrified by more than $50 \%$. Conversely, more than $4 \mathrm{~km}$ below the mother cloud renitrification would result if all particles were to evaporate at this time. Each additional day with $S_{\text {NAT }} \gtrsim 1$ increases the denitrification significantly.

Figure 4 compares the total $\mathrm{HNO}_{3}$ VMR of the base line case (a) with two other relevant cases. Panel (b) refers to 


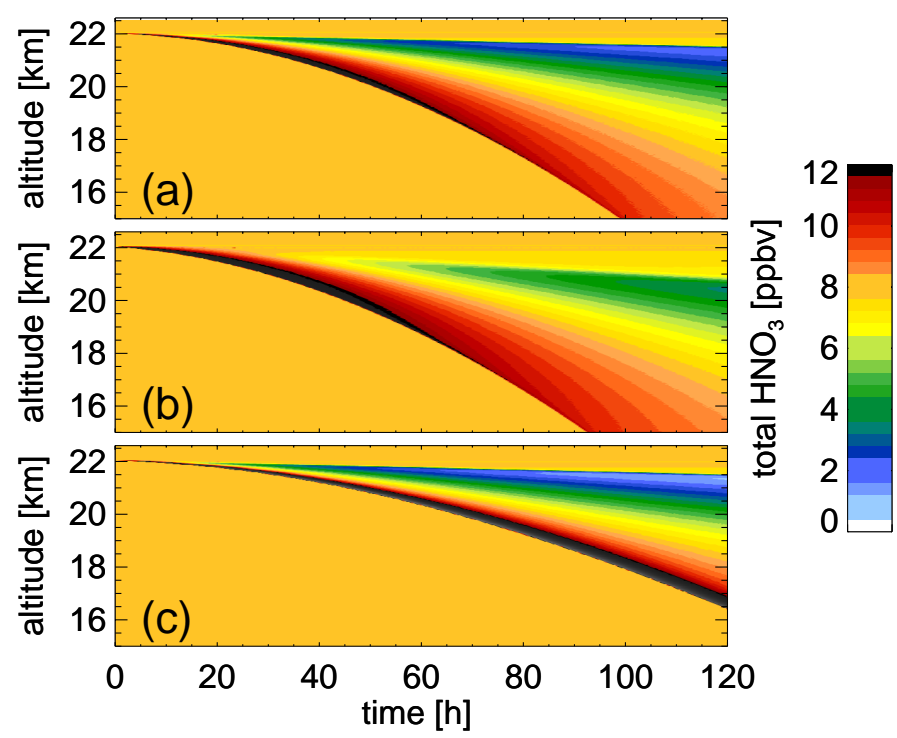

Fig. 4. Total $\mathrm{HNO}_{3}$ (sum of gas phase and particulate). (a) Base line case as in Fig. 2. (b) Mother cloud with $n=0.01 \mathrm{~cm}^{-3}$, all other parameters as in (a). Sedimentation begins earlier, but denitrification is less pronounced than in (a). (c) Total $\mathrm{HNO}_{3}$ for initially $S_{\mathrm{NAT}}=65$ in entire column, which reduces to $S_{\mathrm{NAT}} \approx 30$ due to $\mathrm{HNO}_{3}$ uptake by STS aerosol, all other parameters as in (a). The $\mathrm{HNO}_{3}$-uptake of the STS aerosol delays sedimentation compared to (a).

a less dense mother cloud $\left(n=10^{-2} \mathrm{~cm}^{-3}\right)$. In this case, the FRL does not develop strongly, but the flux conservation regime suffices to reduce number densities to about $10^{-4} \mathrm{~cm}^{-3}$ (see also Fig. 3), yielding a total $\mathrm{HNO}_{3}$ very similar to the base line case. Conversely, increasing the number density within the mother cloud $\left(n=1 \mathrm{~cm}^{-3}\right)$ strengthens the role of the FRL (see also Fig. 3), again leaving the vertical redistribution of $\mathrm{HNO}_{3}$ at lower altitudes unchanged (not shown). Panel (c) shows the effect of supercooled ternary solution droplets (STS) on denitrification. In contrast to panels (a) and (b), here temperatures were chosen such that half of the total $\mathrm{HNO}_{3}$ is taken up by STS, lowering the $\mathrm{HNO}_{3}$ VMR to initially 4 pbbv below the cloud. This leads to approximately halving the sedimentation distance of the NAT particles below the mother cloud and a corresponding slowing of the denitrification process.

\section{Model limitations and sensitivity tests}

Various effects may alter the idealized computations shown above, such as non-monodisperse size distributions, particle shape, gas phase and eddy diffusion, and dynamical effects, e.g. particle dispersion caused by wind shear. A measure of the denitrification strength is the integrated $\mathrm{HNO}_{3}$ mass flux $\int \mathrm{d} z j_{\mathrm{HNO} 3}(z, t)$. Table 1 compares various sensitivity runs in terms of this quantity normalized to the base line result.
Table 1. Denitrification strength $\int j_{\mathrm{HNO} 3}(z, t) \mathrm{d} z$ for $t=3$ days normalized to base line case (Fig. 2) with $\int j_{\mathrm{HNO} 3}(z, t) \mathrm{d} z=$ $14.6 \mathrm{~km}^{2} \mathrm{ppbv} / \mathrm{day}$

\begin{tabular}{lc}
\hline Scenario & $\begin{array}{c}\text { Norm. denitrification } \\
\text { strength }\end{array}$ \\
\hline base line case & 1 \\
aspheric particles (aspect ratio 1:3) & 1.03 \\
lognormal size dist. $(\sigma=1.4)$ & 1.03 \\
without gas diffusion & 0.99 \\
mother cloud with $n=0.01 \mathrm{~cm}^{-3}$ & 1.37 \\
mother cloud with $n=1 \mathrm{~cm}^{-3}$ & 0.91 \\
STS below mother cloud & 0.73 \\
\hline
\end{tabular}

Vertical thickness the of mother cloud. All calculations assume an infinitely thick mother cloud to demonstrate the basic properties of the sedimenting particles. A finite thickness limits the time particles can sediment out of the mother cloud (which then eventually ceases to exist) but does not affect the particle flux, number density or radius of the sedimenting particles. To allow ongoing sedimentation out of the mother cloud for 5 or more days, a thickness of $\gtrsim 12 \mathrm{~m}$, $\gtrsim 68 \mathrm{~m}$, and $\gtrsim 440 \mathrm{~m}$ is required for a mother cloud with $n=1 \mathrm{~cm}^{-3}, n=0.1 \mathrm{~cm}^{-3}$, and $n=0.01 \mathrm{~cm}^{-3}$, respectively.

Effect of particle size distribution. A noteworthy aspect is the insensitivity of denitrification to the initial size distribution. Lognormal and monodisperse distributions yield similar results as the particle number density reduction is dominated by the flux reduction/flux conservation scheme discussed above and not by preferrential sedimentation of initially larger particles. However, a wider distribution of particle sizes in the mother cloud yields also a wider distribution of particle sizes in the sedimenting particle ensemble.

Particle shape. The asphericity of NAT particles affects their sedimentation velocity and growth time. The sedimentation velocity of spherical particles is about $25 \%$ higher than of aspherical particles with an aspect ratio of 1:3, which serves as a case of extreme asymmetry (Müller and Peter, 1992). In addition, this small difference is partly compensated by more rapid growth of $5-10 \%$ of the aspherical particles.

Gas phase and eddy diffusion. Implementation of gas phase diffusion in the model leads to an initially increased $\mathrm{HNO}_{3}$ supply for particles above the cloud base, but changes the results in Figs. 2-4 negligibly. The same holds true for stronger eddy diffusion, although it leads to a more pronounced transport of $\mathrm{HNO}_{3}$ into the mother cloud and smoothing of the entire resulting $\mathrm{HNO}_{3}$ profile.

Dynamical effects. A microphysical column model can- 
not reflect the 3-D wind and temperature fields. Wind shear leads to a distortion of the sedimenting particle ensemble, and we may no expect to observe large NAT-particles directly below type 1a/1a-enh PSCs. Furthermore, during mother cloud formation, e.g. in a mountain lee wave event involving ice particles (Carslaw et al., 1998; Tsias et al., 1999; Voigt et al., 2000), mesoscale variations in NAT particle number density may initially obscure signs of sedimentation, which become apparent only after about one day. In addition, wind shear causes particles to sediment through different airmasses, which increases the volume of air denitrified by the sedimenting particles (Dhaniyala et al., 2002).

\section{Conclusions}

This model study shows that type 1a and type 1a-enh PSCs with $n=0.01-1.0 \mathrm{~cm}^{-3}$ can be a source of NAT-rocks, which efficiently denitrify the polar vortex. Sensitivity studies show that the basic properties of the mother cloud/NATrock mechanism are surprisingly robust against changes in the particle number density, size distribution and shape within the mother cloud, as well as to gas phase or eddy diffusion effects. Large scale transport and wind shear may lead to geographic distortions of the sedimenting NAT-rock population, but neither to enhanced nor reduced denitrification as long as the air remains supersaturated with respect to NAT and mixing processes are not dominant. The resulting NAT-rock number densities and $\mathrm{HNO}_{3}$ vertical flux agree well with in situ measurements. For 20 January, Fahey et al. (2001) estimate the particle number concentration for the larger mode to be $2.3 \times 10^{-4} \mathrm{~cm}^{-3}$ with a mode radius of $7.25 \mu \mathrm{m}$ and a $\mathrm{HNO}_{3}$ flux of $2.6 \mathrm{ppbv} \times \mathrm{km} /$ day. Our model runs in Figs. 2 and 3 show particle number densities to range from $1 \times 10^{-3} \mathrm{~cm}^{-3}$ about $2 \mathrm{~km}$ below the mother cloud to less than $1 \times 10^{-4} \mathrm{~cm}^{-3}$ at lower altitudes. The modelled $\mathrm{HNO}_{3}$ flux a few kilometers below the mother cloud ranges from 1 to $5 \mathrm{ppb} \times \mathrm{km} /$ day, with a maximum of $9 \mathrm{ppb} \times \mathrm{km} /$ day close to the leading edge of the sedimenting NAT-rocks. The model indicates that severe denitrification occurs only if the NAT particles sediment in ambient air with temperatures below the NAT existence temperature for several days. Even in a very cold Arctic winter like the winter 1999/2000 (Manney and Sabutis, 2001), this requirement reduces the number of type $1 \mathrm{a} / 1 \mathrm{a}$-enh clouds which are suited for the development of NAT-rocks.

Acknowledgements. We are grateful to S. Dhaniyala, K. A. McKinney, P. Wennberg, R. S. Gao and D. W. Fahey for fruitful and critical discussions. SF has been supported through the EC-project THESEO-2000/EuroSOLVE (under contract BBW 99.0218-2, EVK2-CT-1999-00047) and through an ETHZ-internal research project.

\section{References}

Biele, J., Tsias, A., Luo, B. P., Carslaw, K. S., Neuber, R., Beyerle, G., Peter, Th.: Nonequilibrium coexistence of solid and liquid particles in Arctic stratospheric clouds, J. Geophys. Res., 106, 22991-23007, 2001.

Browell, E. V., Butler, C. F., Ismail, S., Robinette, P. A., Carter, A. F., Higdon, N. S., Toon, O. B., Schoeberl, M. R., and Tuck, A. F.: Airborne lidar observations in the wintertime arctic stratosphere: polar stratospheric clouds, Geophys. Res. Lett., 17, 385 388, 1990.

Carslaw, K. S., Luo, B. P., and Peter, Th.: An analytic expression for the composition of aqueous $\mathrm{HNO}_{3}-\mathrm{H}_{2} \mathrm{SO}_{4}$ stratospheric aerosols including gas phase removal of $\mathrm{HNO}_{3}$, Geophys. Res. Lett., 22, 1877-1880, 1995.

Carslaw, K. S., Wirth, M., Tsias, A., Luo, B., Dörnbrack, A., Leutbecher, M., Volkert, H., Renger, W., Bacmeister, J. T., Reimer, E., and Peter, Th.: Increased stratospheric ozone depletion due to mountain-induced atmospheric waves, Nature, 391, 675-678, 1998.

Dhaniyala, S., McKinney, K. A., and Wennberg, P. O.: Lee-wave clouds and denitrification of the polar stratosphere, Geophys. Res. Lett., (in press), 2002.

Fahey, D. W., Gao, R. S., Carslaw, K. S., Kettleborough, J., Popp, P. J., Northway, M. J., Holecek, J. C., Ciciora, S. C., McLaughlin, R. J., Baumgardner, D. G., Gandrud, B., Wennberg, P. O., Dhaniyala, S., McKinney, K., Peter, T., Salawitch, R. J., Bui, T. P., Elkins, J. W., Webster, C. R., Atlas, E. L., Jost, H., Wilson, J. C., Herman, R. L., and Kleinböhl, A: The detection of large $\mathrm{HNO}_{3}$-containing particles in the winter Arctic stratosphere, Science, 291, 1026-1031, 2001.

Fueglistaler, S., Luo, B. P., Buss, S., Wernli, H., Voigt, C., Peter, Th., Müller, R., Neuber, R., Hostetler, C., Poole, L., Flentje, H., and Fahey, D. W.: Large NAT particle formation by mother clouds: analysis of SOLVE/THESEO-2000 observations, Geophys. Res. Lett., (in press), 2002.

Gao, R. S., Richard, E. C., Popp, P. J., Toon, G. C., Hurst, D. F., Newman, P. A., Holecek, J. C., Northway, M. J., Fahey, D. W., Danilin, M. Y., Sen, B., Aikin, K., Romashkin, P. A., Elkins, J. W., Webster, C. R., Schauffler, S. M., Greenblatt, J. B., McElroy, C. T., Lait, L. R., Bui, T. P., and Baumgardner, D.: Observational evidence for the role of denitrification in Arctic stratospheric ozone loss, Geophys. Res. Lett., 28, 2879-2882, 2001.

Hanson, D. and Mauersberger, K.: Laboratory studies of nitric acid trihydrate: implications for the south polar stratosphere, Geophys. Res. Lett., 15, 855-858, 1998.

Manney, G. L. and Sabutis, J. L.: Development of the polar vortex in the 1999-2000 arctic winter stratosphere, Geophys. Res. Lett., 27, 2589-2592, 2001

Müller, R. and Peter, Th.: The numerical modelling of the sedimentation of polar stratospheric cloud particles, Ber. Bunsenges. Phys. Chem., 96, 353-361, 1992.

Tabazadeh, A., Jensen, E. J., Toon, O. B., Drdla, K., and Schoeberl, M. R.: Role of the stratospheric polar freezing belt in denitrification, Science, 291, 2591-2594, 2001.

Toon, O. B., Browell, E. V., Kinne, S., and Jordan, J.: An analysis of lidar observations of polar stratospheric clouds, Geophys. Res. Lett., 17, 393-396, 1990.

Toon, O. B., Tabazadeh, A., Browell, E. V., and Jordan, J.: Analysis of lidar observations of Arctic polar stratospheric clouds during January 1989, J. Geophys. Res., 105, 20 589-20 615, 2000. 
Tsias, A., Wirth, M., Carslaw, K. S., Biele, J., Mehrtens, H., Reichardt, J., Wedekind, C., Weiss, V., Renger, W., Neuber, R., Von Zahn, U., Stein, B., Santacesaria, V., Stefanutti, L., Fierli, F., Bacmeister, J., and Peter, Th.: Aircraft lidar observation of an enhanced type 1a polar stratospheric cloud during APE-POLECAT, J. Geophys. Res., 104, 23 961-23 969, 1999.

Voigt, Ch., Schreiner, J., Kohlmann, A., Zink, P., Mauersberger, K., Larsen, N., Deshler, T., Kröger, Ch., Rosen, J., Adriani, A., Cairo, F., Di Donfrancesco, G., Viterbini, M., Ovarlez, J., Ovar- lez, H., David, Ch., and Dörnbrack, A.: Nitric acid trihydrate (NAT) in polar stratospheric clouds, Science, 290, 1756-1758, 2000.

Waibel, A. E., Peter, Th., Carslaw, K. S., Oelhaf, H., Wetzel, G., Crutzen, P. J., Pöschl, U., Reimer, E., and Fischer, H.: Arctic ozone loss due to denitrification, Science, 283, 2064-2069, 1999.

WMO: Scientific Assessment of Ozone Depletion: 1998, Rep. 44, World Meteorological Organization, Geneva, Switzerland, 1999. 